\title{
Aproximaciones analíticas al campo estadístico de la violencia. Avances de investigación
}

\author{
María Belén Muñiz y Cristian Tibaldi \\ belumuniz@hotmail.com
}

\section{Presentación}

Desde múltiples lugares nos llegan a diario las voces que denuncian, lamentan y señalan un aumento de la violencia "callejera" que atraviesa a las y los jóvenes, colocándolos mucho más en el lugar de ejecutores que de víctimas de dicha violencia. Pero más allá de la atribución de responsabilidad, que sobre estos jóvenes se puede dar desde los diferentes sectores de la sociedad donde se origina la demanda, observamos una necesidad permanente de cuantificar, o por lo menos de poner en término de magnitudes relativas la intensidad de dicha violencia. Esta parte singular de la demanda por intervenir sobre la violencia, ya sea desde discursos de endurecimiento penal que piden mayor presencia policial y mayor castigo (Garland, 1990), o desde aquellos que se estructuran en torno al pleno ejercicio de los derechos humanos y la inclusión social, nos ha conducido a preguntar si es posible medir la violencia. ¿Qué información debe ser tomada como relevante a la hora de afirmar el aumento o disminución de la violencia?, ¿existe un desarrollo objetivo y autónomo de los hechos violentos que se pueda expresar en magnitudes relativas o, por el contrario, es efecto de un discurso producto de una "sensación" que varía en función del sector social que lo enuncie?

Inmediatamente nos detenemos en este punto: ¡es sólo un agrupamiento de hechos más o menos conexos o se inscriben en una secuencia, un proceso social de larga duración?, ¿cómo se nos presenta la información sobre los hechos de violencia que nos permita arrojar luz sobre estas cuestiones?

En este ensayo presentaremos algunos avances de la investigación que los autores llevamos adelante en torno a la temática de la violencia, en el marco de un proyecto dedicado a indagar las diferentes formas en que las y los jóvenes son atravesados por prácticas y situaciones donde la violencia se ejerce en sus mismos cuerpos. Esto último sobre la definición conceptual que concibe al cuerpo como un particular "territorio en que se expresan confrontaciones entre conjuntos de relaciones sociales" (Forte y Pérez, 2010, p. 12), punto nodal donde las tensiones que estructuran al capitalismo como formación socio-histórica toman su materialidad. Asimismo, para abordar la temática de la violencia tomamos como definición conceptual la otorgada por la Organización Mundial de la Salud (OMS), que la caracteriza como "El uso intencional de la fuerza o el poder físico, 


\section{2/ Perspectivas Metodológicas /19/Vol. II /Año 2017}

de hecho o como amenaza, contra uno mismo, otra persona o un grupo o comunidad, que cause o tenga muchas probabilidades de causar lesiones, muerte, daños psicológicos, trastornos del desarrollo o privaciones" (OMS, 2002, p. 3).

La concepción del cuerpo como territorialidad implica necesariamente entenderlo como producto de procesos históricos sociales de largo alcance y a la vez campo de confrontación de fuerzas sociales (Núnéz, 2012; Marín, 1996; Forte y Pérez, 2010). Desde el advenimiento de la Modernidad, el cuerpo fue consolidándose como objeto de gestión, y de las tecnologías de poder desplegadas a tal efecto (Foucault, 1987), bajo la forma en apariencia neutrales de las políticas públicas. El control sobre poblaciones socialmente peligrosas, moral y materialmente riesgosas, va instalando en los diferentes niveles del poder prácticas que buscarán disciplinar, ordenar, contener y eventualmente suprimir dichas fuentes de riesgo (Daroqui, López y García, 2012). La juventud se ha ido transformando cada vez con mayor frecuencia en una fuente de riesgo social -mucho más aún si está unida a la pobreza- y atravesada por dos discursos que reactualizan, paradójicamente, el estigma: uno de corte asistencialista en el cual se reconoce la vulnerabilidad de las y los jóvenes, que dispara una serie de dispositivos de promoción y protección de derechos que buscan "salvar" a dichos sujetos de la delincuencia, las drogas y el desempleo. Por otro lado, un discurso que estigmatiza y responsabiliza a las y los jóvenes por el aparente aumento de hechos delictivos, sensación de inseguridad y expansión del narcotráfico, creando articuladamente con el primero todo un cuerpo legal-jurídico de carácter punitivo que busca neutralizar esa fuente de riesgo social.

Este ensayo presenta la primera etapa del proyecto que refiere a la elaboración y análisis de una base de datos sobre las fuentes más visibles de información, registrando el contenido de todos los sitios web donde las diferentes agencias estatales y no estatales publican datos de acceso público sobre hechos de violencia referidos al aglomerado Mar del Plata/Batán. El recorte correspondiente a la franja etaria que elegimos se fue adaptando a la cantidad y calidad de la información disponible. Esta elección metodológica, en apariencia menor, fue consecuencia directa de las características propias de la información que fuimos encontrando, por el hecho de que la mayoría de los datos analizados están relacionados en mayor o menor medida con la conflictividad con la ley penal. En efecto, de 24 fuentes consultadas 20 corresponden a fuentes judiciales, policiales o relacionadas con el Sistema de Responsabilidad Penal Juvenil (SRPJ), lo que nos llevó a fijar al grupo etario objetivo entre los 15 y los 21 años de edad, correspondiente en su límite inferior con un año antes de la edad de punibilidad, que según la Ley 26061 es a partir de los 16 años, y en su límite superior con la categoría "jóvenes adultos", entre 18 y 21 años, que establece la Ley 12256 de Ejecución Penal. Esto no significa que exista una correspondencia entre este grupo etario y los grupos etarios de las fuentes. Muy por el contrario, existe una gran variabilidad a la hora de fijar la categoría "joven" y en muchas fuentes se toman hasta los 24 o incluso 26 años, lo que vuelve a veces incomparable dicha información.

\section{Análisis descriptivo de las fuentes disponibles de acceso público}

Acerca de la información publicada por la Procuración General de la Suprema Corte de Justicia de la Provincia de Buenos Aires, debemos advertir que refiere siempre a las 
María Belén Muñiz y Cristian Tibaldi / Aproximaciones analíticas al campo[...] 93 Investigaciones Penales Preparatorias (IPP), lo cual significa que son números que no hablan de personas sino de causas, en las cuales puede haber más de un imputado o, inversamente, un sujeto imputado en más de una causa.

En el caso del informe Caracterización de homicidios dolosos y robos con armas, estudio descriptivo de IPP, la información publicada sólo desagrega del total de la provincia tres grandes grupos de Departamentos Judiciales: Conurbano, Grandes (entre los que se encuentra el Depto. Judicial Mar del Plata) e Interior, con lo cual no es posible construir datos específicos para el territorio estudiado. Se puede señalar como dato relevante que para el año 2013, con una población 723.685 habitantes, para 89 IPP's iniciadas que involucraron a 91 fallecidos, la tasa (víctimas de homicidios dolosos consumados-HDC cada 100 mil habitamtes) fue de 12,57, superada sólo por Quilmes: 12,75. La inmediata inferior fue La Matanza, con 11,49.

De los informes publicados sobre el Fuero de Responsabilidad Penal Juvenil (FRPJ), el que más se acerca a nuestras necesidades es el referido a IPP FRPJ iniciadas por Bien J. y por Depto. Judicial, correspondiente al período 2012-2014. El resto de los informes, a saber: IPP FRPJ iniciadas por Depto. Judicial (2009-2014), IPP FRPJ iniciadas por Bien Jurídico Protegido total PBA (2009-2014) e IPP FRPJ iniciadas por Partido (2012) no contienen información suficientemente desagregada.

En relación al Ministerio de Seguridad de la Provincia de Buenos Aires, sólo nos provee de estadísticas criminales en informes presentados desde el 2010 hasta el 2012. Esta información provista por el Ministerio utiliza como fuente de análisis cuantitativo los datos contenidos en la base SIMP de la Procuración General de la Suprema Corte de Justicia de la Provincia, confrontándolos con los datos emanados de las bases de información del Ministerio de Justicia y Seguridad (desde el año 2013 separados en Ministerio de Seguridad y Ministerio de Justicia). Los datos son prácticamente los mismos que los publicados por la Procuración General, pero se completan los análisis con elaboración propia sobre registros del propio Ministerio. Pese a que esta información es de nuestra incumbencia, la misma no se encuentra lo suficientemente detallada y desagregada; vale decir, no es información completa ni exhaustiva al no encontrarse analizada con respecto a la edad ni al territorio. La única información que provee sobre el territorio consiste en separar el conurbano bonaerense del resto de la Provincia de Buenos Aires en relación tanto a la participación en las estadísticas criminales como en el núcleo de delitos vinculados a la seguridad ciudadana. Por otra parte, el Ministerio de Seguridad publica el informe Situación de las personas privadas de libertad en la Provincia de Buenos Aires. Esta consiste en un informe que abarca el periodo de Diciembre 2007 a Octubre 2011. Al igual que las estadísticas criminales, esta información toma como fuente a la Corte Suprema de Justicia de la provincia, por lo que toda la información es analizada sobre el total de la provincia salvo algunos pocos datos. Entre ellos, sólo encontramos información desagregada para la Unidad 15 de Mar del Plata-Batán, en lo que refiere a la cantidad de plazas habilitadas en el marco del programa "Casas por Cárceles" y la ocupación de las mismas al 15 de septiembre de 2011.

En lo que respecta al Centro Municipal de Análisis Estratégico del Delito, nos encontramos con que es el único organismo estatal que nos provee de información espe- 


\section{4/ Perspectivas Metodológicas /19/Vol. II /Año 2017}

cífica sobre la ciudad de Mar del Plata. Esta agencia estatal posee informes estratégicos de la ciudad en relación a robos y hurtos de automotores (2014-2015); violencia de género: análisis de denuncias penales (2014); informes sobre homicidios dolosos (20132014) y por último informes sobre tenencia de estupefacientes para consumo personal (2014). A su vez, nos provee de informes semanales y mensuales específicos de análisis criminal sobre el funcionamiento del Comando de Prevención Comunitaria (CPC) y de georreferencia del comportamiento delictual durante el año 2014 y 2015. Publican además una síntesis del análisis estratégico de las primeras 18 semanas (6 de junio al 9 de octubre de 2014) de funcionamiento del Comando de Prevención Comunitaria del partido de Gral. Pueyrredón. En relación a nuestro objeto de estudio, la única información de utilidad directa es la relacionada tanto con este último informe como con la tenencia de consumo de estupefacientes y la de homicidios dolosos, por ser los únicos datos que se encuentran relacionados con la edad. El Informe sobre las 18 semanas de funcionamiento del CPC nos brinda información sobre la cantidad de aprehendidos por esta fuerza menores de 18 ańos y el motivo de la aprehensión, comparando las semanas desde el 6 de junio al 9 de octubre del 2014. Con respecto al motivo de la aprehensión por robo con o sin arma de fuego y por hurto, observamos que en todos los casos el grupo de varones de más de 18 años fue el más numeroso. Salvo en la semana 3, en la que hubo una cantidad inédita, esto es, 32 aprehendidos, de los cuales 14 (43,7 \%) fueron varones menores de 18 ańos aprehendidos por robos con y sin arma de fuego. Pese a ello, en el resto del período la proporción de esta franja etaria ha sido más pequeña. En el caso del hurto es considerablemente mayor la cantidad de aprehendidos menores de 18 ańos varones, en varias semanas más que en el caso del robo. Es más notorio (aunque menor con respecto a la cantidad general) el involucramiento de mujeres -en mayor proporción la cantidad de mujeres mayores de 18 ańos-. Asimismo, en el caso de los aprehendidos por el CPC por averiguación de identidad o ilícito observamos, por la misma cantidad de semanas, que también sucede que los varones mayores de 18 años mantienen los niveles más altos. En cuanto a los menores de edad, se mantiene en importante menor proporción que los aprehendidos mayores, al tiempo que durante cuatro semanas no hubo aprehendidos menores. Por último, en cuanto a los aprehendidos por encubrimiento, si bien la mayoría son aprehendidos mayores de edad -como sucede en todos los motivos de aprehensión- llama la atención el número alto de aprehendidos menores de 18 años, muy superior a la media de todos los demás casos.

Por otro lado, tanto el informe sobre los procedimientos realizados por la Policía Bonaerense de Seguridad vinculados con los casos de infracción a la Ley 23.737 -tenencia por estupefacientes- durante el año 2014, como el Informe sobre homicidios dolosos (2013-2014), utilizan como fuentes de datos la base proporcionada por el CEPAID (Centro de Procesamiento de Análisis Informático Delictual del Ministerio de Seguridad de la Provincia de Buenos Aires). Entre el 1 de enero y el 31 de diciembre de 2014, el CEPAID reportó 1.035 casos que tenían relación directa con infracciones a la Ley 23.737. De esos casos, 756 fueron por tenencia para consumo personal; de estos últimos 666 tuvieron por objeto la sustancia estupefaciente marihuana; a su vez, en esos 666 casos fueron aprehendidas 1.075 personas por tenencia de marihuana, de los $111(10,33$ $\%)$ fueron varones menores de 18 años y $2(0,19 \%)$ fueron mujeres menores. Por último, el Informe de homicidios dolosos (2013-2014), además de tomar como punto de partida para su análisis los datos emanados del CEPAID, utilizaron como complemento 
María Belén Muñiz y Cristian Tibaldi / Aproximaciones analíticas al campo[...] 95 datos provenientes del Sistema Informático del Ministerio Público Fiscal (SIMP), datos de procedimientos del Comando de Prevención Comunitaria General Pueyrredón, con el relevamiento de su cobertura por los medios de comunicación y cifras parciales provenientes del sistema de salud. Entre el 1 de enero del 2013 y 31 de diciembre del año 2014, se registran 74 homicidios dolosos, con 76 personas fallecidas en el área del Municipio de General Pueyrredón, de los cuales más de un 90\% fueron hombres. En el año 2013 un $27 \%$ de las víctimas tenían entre 16 y 25 años de edad. Asimismo, el 50\% de las víctimas de homicidios dolosos ocurridos en 2013 tenían entre 16 y 35 años. En el año 2014, un 22\% de las víctimas tenían entre 16 y 25 años y un $47 \%$ perteneció a la franja etaria de 16-35 años.

La Secretaría de Niñez y Adolescencia de la Provincia de Buenos Aires (SNyA) publica información relevante por medio del Registro Estadístico Unificado de Niñez y Adolescencia (R.E.U.N.A.). Este sistema permite hacer consultas online sobre número de NNyA abordados anualmente por territorio (que indica el Servicio Zonal de Promoción y Protección de Derechos -SZPPD- en nuestro caso Mar del Plata); por motivo de la intervención; por organismo (el efector que solicitó intervención al SZPPD, es decir, por pedido de quién); por género y finalmente por edad. El sistema permite realizar cruces entre dichos ítems y solicitar información complementaria vía correo electrónico. Al día de publicación de este trabajo, la repartición no contestó los pedidos hechos por el equipo de investigación.

La información publicada permite un acercamiento a la cantidad de menores que pasan por el Sistema de Promoción y Protección de Derechos (SPPD), con la salvedad de que se registran las entradas al mismo pero no la evolución de cada caso; de modo que un niño o niña puede ser abordado una sola vez por algún operador, volver a la situación que originó la consulta, quedar asentada esa entrada en el sistema pero no tener un efecto real sobre el sujeto vulnerado. Esta situación es similar al caso de los datos de Procuración: se puede formar una IPP por un delito, pero no se puede saber cómo evoluciona esa causa, si fue archivada, si llegó a juicio, si hubo sobreseimiento o, por el contrario, se ejecutó una sentencia, cuántas medidas alternativas al encierro se solicitaron y cuántas se aplicaron, entre otras variables que permitirían conocer mejor.

La Municipalidad de General Pueyrredón dispone de una página web desde donde se puede ingresar al ítem Estadísticas. Allí sólo se reproducen datos copiados del INDEC y de la Dirección Provincial de Estadísticas del Ministerio de Economía de la Provincia de Buenos Aires, que en el ítem Seguridad replica datos del Ministerio de Justicia y Seguridad de la PBA, ya analizados arriba.

El Observatorio Social Legislativo, dependiente de la Honorable Cámara de Diputados de la PBA, publica desde el año 2010 el informe anual sobre adolescencia $\mathrm{La}$ adolescencia $M I D D E$. En dicha publicación existe un capítulo para la Dimensión Violencia contra NNA y otro para la Dimensión Justicia Penal. La información no está desagregada por partido, sino que agrupa el total de la PBA y son datos elaborados sobre cifras del Ministerio de Salud y Sistema SIMP del Ministerio Público Fiscal. A partir del 2014, publica el informe La niñez MIDDE, con las mismas fuentes de información y, por ende, los mismos niveles de desagregación insuficiente de categorías. 
El resto de las fuentes consultadas, como la Estadística de muertes en el Servicio Penitenciario, publicada por el Ministerio de Seguridad de la PBA, cifras del Ministerio de Salud de la Provincia de Buenos Aires, información de La Procuración Penitenciaria de la Nación junto con el Comité Contra la Tortura y el Grupo de Estudios sobre Sistema Penal y Derechos Humanos (Registro Nacional de casos de tortura y/o malos tratos del año 2012), son fuentes que eventualmente hacen referencia a alguna de las variables en estudio (territorio y edad), pero poco detallada y sin suficiente grado de desagregación.

\section{Consideraciones finales}

La mayoría de la información analizada no se encuentra actualizada, siendo prácticamente nula la información sobre 2015, al tiempo que prima una gran variabilidad en los límites temporales. A su vez, la mayoría de las fuentes se originan en agencias de control social penal, como el Poder Judicial y el Ministerio Público Fiscal, lo cual muestra el protagonismo que la dimensión legal-punitiva tiene por sobre otros aspectos de la violencia. Este factor puede tener como resultante la primacía de cifras enmarcadas en la relación conflictiva que los jóvenes tienen con la ley, relegándose el registro de cifras sobre procesos de violencia. Gran parte de los datos encontrados reflejan lo antedicho. Este profundo problema social -en lo que al registro de los hechos que lo conforman como proceso se refiere- se ha convertido paulatinamente en una mera tarea técnica encargada a personal con poca formación en el área metodológica, a la vez que el diseño estadístico se da profundamente ligado a las necesidades políticas del momento. Una de las mayores consecuencias de ello es la invisibilidad de los sujetos implicados en un hecho de violencia. La mayor parte de la información encontrada refiere a hechos, a datos presentados y analizados como una fotografía instantánea, dejando invisibilizado el conjunto de acciones que los cuerpos viabilizan, vale decir, las relaciones sociales que hay por detrás del hecho. Esto mismo lo cristaliza el análisis de las fuentes realizado con antelación. Sólo dos de las 24 fuentes analizadas -CeMAED y R.E.U.N.A.- nos provee información sobre las tres variables objeto de estudio: violencia, edad y territorio. Esto es, cierta información específica de un contexto social con particularidades propias, relacionando el hecho violento registrado con características del sujeto víctima y/o victimario. El resto de las fuentes construye las cifras de violencia como hechos puntuales y aislados de todo proceso social, los cuales permiten caracterizar los efectos de la violencia pero sin ahondar en las causas que la generan a nivel social e institucional.

La carencia de información confiable y comparable en el tiempo vuelve la publicación de estadísticas más un ejercicio de propaganda institucional que un registro útil para conocer la evolución -es decir, la manera en que se dan los cambios- de los procesos que instituyen relaciones sociales violentas. Entendemos sin embargo la importancia de comenzar por un diagnóstico de la realidad basado en datos estadísticos que confronten el ámbito de las sensaciones y especulaciones con hechos empíricos. Pero, al mismo tiempo, nos parece imprescindible comenzar a profundizar esta forma de construir y analizar la estadística, focalizando la mirada en el seguimiento del devenir punitivo, en este caso en la trayectoria de la violencia. Lo que proponemos entonces es comenzar a construir sistemas integrales de información, mapeos y rutas que abarquen todas las instancias por las cuales transita un joven protagonista de un hecho de violencia. 
María Belén Muñiz y Cristian Tibaldi / Aproximaciones analíticas al campo[...] 97

\section{Bibliografía}

- Daroqui, A., López, A. L. y García, R. (2012). Sujeto de castigos: hacia una sociología de la penalidad juvenil. Buenos Aires: Homo Sapiens

- Forte, G. y Pérez, V. (2010). El cuerpo, territorio del poder. Buenos Aires: Colectivo Ediciones/P.I.Ca.So.

- Foucault, M. (1987). Historia de la sexualidad III. La inquietud de sí. México, D. F.: Siglo XXI.

- Garland, D. (1990). Castigo y sociedad moderna. Un estudio de teoría social. México, D. F.: Siglo XXI.

- Marín, J. C. (1996). Conversaciones sobre el poder (Una experiencia colectiva). Buenos Aires: Archivo Chile.

— Núnez, A. (2012). Lo que el agua (no) se llevó. Política urbana: poder, violencia e identidades sociales. Buenos Aires: El Colectivo.

— Organización Mundial de la Salud (2002). Informe Mundial sobre la Violencia y la Salud. Ginebra. Recuperado de http://apps.who.int/iris/bitstream/10665/67411/1/ a77102_spa.pdf 